\title{
Competencias clave para la empleabilidad de los egresados universitarios: Un análisis en la Universidad de Zaragoza
}

\author{
Rosaura Villarroya Martín \\ UNIVERSA \\ UNIVERSIDAD DE ZARAGOZA \\ rosavil@unizar.es \\ Pedro José Ramos-Villagrasa \\ Departamento de Psicología y Sociología \\ UnIVERSIDAD DE ZARAGOZA \\ pjramos@unizar.es
}

\section{Resumen:}

Introducción: La investigación sobre factores que favorecen la empleabilidad es muy importante hoy día. En este sentido, la formación universitaria siempre se ha considerado un valor entre los jóvenes, pero para desempeñar un puesto de trabajo son necesarias tanto competencias específicas del puesto como otras genéricas. El presente estudio hace énfasis en estas últimas, teniendo como 
objetivo determinar cuáles son las competencias genéricas que se relacionan con la empleabilidad de los titulados universitarios.

Material y métodos: 1.174 participantes, entre estudiantes de último curso y recién egresados de la Universidad de Zaragoza, fueron evaluados en una serie de competencias al finalizar sus prácticas en empresas y organismos a través de UNIVERSA, el Servicio de Orientación y Empleo de la citada Universidad. Las evaluaciones fueron realizadas por los profesionales que ejercieron de tutores en la organización donde realizaron sus prácticas y posteriormente se analizaron utilizando estadística descriptiva y análisis de diferencia de medias.

Resultados: Los participantes contratados destacan por un mayor rendimiento, autonomía, seguridad en sí mismos y madurez, e iniciativa. En cambio, los participantes a los que la empresa recomienda no contratar son peor valorados en todas las competencias. Por último, se observa que las mujeres puntúan significativamente más alto en presencia física que los hombres.

Discusión: Los resultados obtenidos ofrecen algunas pautas sobre las competencias que pueden requerir apoyo por parte de los servicios de orientación para incrementar la empleabilidad de los estudiantes. Después, se destacan las principales limitaciones y se proponen ideas de investigación futura.

Palabras clave: Competencias, empleabilidad, egresados universitarios, prácticas, búsqueda de empleo.

\section{Key Skills for the Employability of University Graduates: an Analysis at the University of Zaragoza}

\section{Abstract:}

Introduction: research into the factors favouring employability is very important nowadays. In this regard, university education has always been looked upon as a value by young people but in order to obtain a post it is necessary to have both job-specific and also more generic skills. This paper highlights the latter, and aims to determine the generic skills relating to employability for university graduates. Material and methods: 1,174 participants, including undergraduates 
in their final year of study and recent graduates from the University of Zaragoza, were assessed in a number of competences upon completion of their practicals in companies and organisations through UNIVERSA, the Career Guidance Service of the aforementioned university. The assessments were conducted by professionals who acted as tutors in the organisation where the students did their practicals and were then analysed using descriptive statistics and mean difference analysis.

Results: the participants contracted stand out for their high level of performance, autonomy, self-confidence and maturity, and initiative. In contrast, participants that the company does not recommend hiring for work are not valued so highly in all skills. Finally, it is observed that women score significantly higher in physical presence than men.

Discussion: the results obtained offer some guidance on the skills that might need support from the career guidance services to increase students' employability. Attention is then drawn to the main limitations, and ideas for future research are proposed.

Key words: Competencies, employability, university graduates, practicals, search for employment. 



\section{Competencias clave para la empleabilidad de los egresados universitarios: Un análisis en la}

Rosaura Villarroya Martín Universidad de Zaragoza

Pedro José Ramos-Villagrasa

\section{Agradecimientos}

El presente artículo ha sido posible gracias a la colaboración del Servicio de Orientación y Empleo UNIVERSA y se ha desarrollado a partir de un Trabajo de Fin de Grado elaborado por la primera autora bajo la dirección del segundo.

Pedro J. Ramos-Villagrasa pertenece al Grupo Estudios Sociedad del Riesgo, equipo de investigación consolidado del Gobierno de Aragón (ref. S45). Su participación en este artículo está apoyada por el proyecto de I+D+i con ref. PSI2013-44854-R.

En los tiempos actuales, en los que la Crisis económica lleva aparejada la escasez de puestos de trabajo y las organizaciones se ven sujetas a cambios constantes que les hacen buscar profesionales capaces de desempeñarse en muy distintas situaciones, la investigación sobre factores que favorezcan la empleabilidad cobra especial relevancia. Yorke (2006) define la empleabilidad como el conjunto de características personales que incrementan la capacidad de un individuo para conseguir un empleo en la profesión que ha elegido desempeñar. En este sentido, la formación universitaria siempre se ha tenido como un 
valor en el que confiar para incrementar las probabilidades de obtener un empleo, especialmente entre los jóvenes. Esta idea se ve refrendada por Valero (2012), el cual encuentra que siete de cada diez egresados consigue un trabajo acorde a su formación, aunque sus condiciones de trabajo hayan empeorado respecto a estudios anteriores. Este autor también pone de manifiesto que, junto a los conocimientos académicos, son las competencias transversales adquiridas a lo largo de los estudios las que contribuyen a la empleabilidad de los universitarios. En este artículo pretendemos seguir esta línea, analizando qué competencias transversales contribuyen a la empleabilidad de los egresados de la Universidad de Zaragoza (España).

\section{Las competencias en el ámbito educativo y el laboral}

Las competencias pueden definirse como el producto de características subyacentes a las personas (i.e. conocimientos, habilidades y aptitudes, Forrier, Sels y Stynen, 2009) que se relacionan causalmente con el desempeño en un puesto y organización concretos (Boyatzis, 1982).

El término 'competencia' ha sido muy utilizado en los últimos años, tanto en el ámbito educativo como en el laboral. En el ámbito educativo podemos remontarnos al planteamiento del Espacio Europeo de Educación Superior (EEES), desarrollado a través de la Declaración de Bolonia (1999), la Declaración de Praga (2001), la Reunión del Consejo Europeo (2000) y la Declaración de Lisboa (2007). El EEES es concebido como un proceso de armonización educativa en toda la Unión Europea. El EEES busca pasar de una educación superior centrada en la enseñanza de conocimientos por parte del profesorado a una educación centrada en el aprendizaje de competencias por parte de los estudiantes. Esto es, el EEES quiere desarrollar un modelo de enseñanza funcional donde la principal función del profesor universitario sea posibilitar, facilitar y guiar al alumno para que pueda desarrollar las competencias, contenidos y prácticas profesionales de una determinada disciplina (Herrera, 2006). A partir de aquí es donde se pide a las Universidades que reformen sus currículos en colaboración con los empresarios, que expresen con claridad las competencias adquiridas por los graduados y 
mejoren la empleabilidad de sus egresados (Hué, 2008), labor en la que en cierto modo todavía se encuentran envueltas.

Paralelamente, el concepto de competencia se ha afianzado en el ámbito laboral. De acuerdo con Pereda y Berrocal (2001), las competencias se traducen en comportamientos observables y pueden ser de dos tipos: (1) genéricas, esto es, comunes a todos los puestos de la organización; y (2) específicas, apropiadas para un puesto de trabajo concreto. Esta clasificación es muy similar a la que el proyecto Tuning (s.f.) realiza en el ámbito educativo, entendiendo la existencia de competencias genéricas (transversales), útiles en muy diversos contextos, y específicas de la titulación estudiada.

A priori, podría suponerse que las competencias específicas son las principales e indiscutibles protagonistas del proceso formativo y el posterior desempeño en el puesto de trabajo. Sin embargo, el mercado laboral actual está sujeto a rápidos cambios que exigen a los profesionales ser capaces de adaptarse y desenvolverse en diferentes situaciones (Goodwin, Burke, Wildman y Salas, 2008; Marques-Quinteiro, Ramos-Villagrasa, Passos y Curral, 2015). Además, desde las organizaciones se señala la inquietud ante una cierta carencia por parte de los candidatos de competencias genéricas de tipo social (soft skills), tales como competencias interpersonales o de comunicación (c.f. CEDEFOP, 2014). En este sentido, según datos del Observatorio de Empleabilidad y Empleo Universitarios (OEEU, 2015), entre los requisitos exigidos para optar a un puesto de trabajo las competencias específicas tienen un peso del $32,49 \%$ mientras que las genéricas tienen un peso del $33,49 \%$, es decir, las competencias genéricas se encuentran prácticamente al mismo nivel que las específicas, a las que siempre se les ha dado mayor protagonismo.

Todas estas circunstancias hacen críticas las competencias genéricas. La Universidad, en tanto que contribuye a la formación de los futuros profesionales (González y González, 2008), comparte la responsabilidad de desarrollar éstas en sus egresados, y no solamente las competencias específicas. Por ejemplo, la investigación ha confirmado la relevancia de competencias genéricas como el trabajo en equipo (García-Izquierdo, García-lzquierdo y Ramos-Villagrasa, 2007), si bien la educación superior 
todavía tiene que mostrar cómo proporcionar a los futuros profesionales las habilidades prácticas para cohesionar un equipo y gestionar los procesos del equipo (Scott-Ladd y Chan, 2008).

Volviendo al ámbito universitario, desde el punto de vista práctico resulta fácil recomendar a los estudiantes que se centren en el desarrollo de las competencias específicas, pues éstas se encuentran muy bien acotadas dentro del conjunto de puestos de trabajo usualmente desempeñados por estudiantes de una determinada titulación. En cambio, las competencias transversales son heterogéneas por naturaleza, y no existe consenso sobre cuáles son las más eficaces para incrementar la empleabilidad. Bien es cierto que experiencias como las realizadas por las Universidades de Granada (Valero, 2012), A Coruña (Freire, 2008) y La Laguna (García, 2008) apuntan a la motivación por el trabajo, la responsabilidad, la capacidad de trabajo en equipo, la resolución de problemas y el aprendizaje continuo como las competencias clave que promueven la empleabilidad. Sin embargo, debemos tener en cuenta que cada Universidad se desenvuelve en un contexto autonómico diferente, siendo éste el mercado que suele absorber a la mayor parte de titulados. Al ser contextos diferentes, cabe la posibilidad de que las competencias necesarias para desenvolverse en ellos también lo sean. Esto hace sumamente relevante el estudio de cada contexto de manera aislada para, posteriormente, tratar de establecer reglas generales. Por ello, en este artículo nos hemos propuesto estudiar la situación en Aragón a través de la información existente en el Servicio de Orientación y Empleo de la principal institución universitaria pública de la Comunidad Autónoma, la Universidad de Zaragoza.

\section{UNIVERSA, el Servicio de Orientación y Empleo de la Universidad de Zaragoza}

Como consecuencia de la evolución del sistema educativo universitario y la demanda de compatibilizar el desarrollo de competencias profesionales con la formación científica (Lantarón, 2013), muchas universidades han creado servicios de orientación y empleo que sirvan de refuerzo a esta actividad. 
Existen numerosos estudios que ofrecen una visión general de los distintos servicios de orientación que existen en las Universidades españolas, tanto de su organización como de las distintas actuaciones que llevan a cabo. En nuestro caso, siguiendo a Lantarón (2013) y Salmerón (2010), enunciaremos sus características comunes: (1) se trata de servicios "jóvenes", surgiendo la mayor parte en la década de 1990-2000, coincidiendo con la evolución y expansión de las Universidades españolas; (2) la denominación de todos ellos en las Universidades es muy amplia y heterogénea, incluyendo algún término con el que se muestra que trabajan en la línea del empleo (e.g. Centros de Orientación e información para el Empleo o Servicio Universitario de Empleo); (3) no se trata de servicios autónomos sino que suelen depender o bien del Vicerrectorado de Estudiantes o de Fundaciones Universidad-Empresa; y (4) sus líneas de actuación son variadas, incluyendo la orientación e información, la gestión de prácticas académicas y voluntarias, la gestión de bolsas de empleo, la actuación como agencias de colocación, la realización de actividades de formación, la promoción del emprendimiento y el desarrollo de observatorios ocupacionales.

En el caso de la Universidad de Zaragoza, su Servicio de Orientación y Empleo, denominado UNIVERSA, nace en 1997 y se financia a través del convenio firmado entre el Instituto Aragonés de Empleo (INAEM) y la Universidad. Tal y como muestra su página web (www.unizar.es/universa), su objetivo es favorecer la inserción laboral de los jóvenes universitarios, adecuando su perfil profesional a las necesidades de las empresas. Este objetivo se desarrolla a través de diferentes líneas de trabajo: (1) orientación a los estudiantes y egresados en la búsqueda de empleo, donde se ofrece además de una atención individualizada y personalizada, la posibilidad de realizar actividades formativas en aspectos clave para su inserción laboral como: talleres de búsqueda de empleo y desarrollar competencias profesionales como trabajo en equipo, cómo hablar en público, toma de decisiones, inteligencia emocional, etc.; (2) gestión de actividades formativas, dirigidas a titulados recientes en desempleo, como formación complementaria a sus titulaciones, tanto en habilidades transversales como en materias específicas relacionadas con sus titulaciones; (3) fomento del espíritu emprendedor de los estudiantes, realizando acciones 
de sensibilización y difusión para la creación de empresas y/o autoempleo, talleres de fomento de la actividad emprendedora e información y orientación empresarial; (4) obtención de información, mediante un observatorio de empleo que pretende que las instituciones y profesionales relacionados con el empleo y la formación superior en Aragón dispongan de los datos precisos para tomar decisiones en sus ámbitos de trabajo; y (5) inserción laboral, a través de la gestión de prácticas para estudiantes de último curso, proyectos fin de carrera, así como prácticas ocupacionales nacionales e internacionales para jóvenes titulados en desempleo. En este estudio nos centraremos en esta última línea de actuación, por lo que será descrita con mayor detalle a continuación.

UNIVERSA gestiona una bolsa de organizaciones con sede en Aragón interesadas en acoger estudiantes y recién egresados en prácticas. Las organizaciones deben firmar un convenio en el que se recoge de manera general las condiciones de la colaboración. Posteriormente, se les añade un anexo con las características de las prácticas concretas que se realizarán, dado que éstas pueden ser de muy diversa naturaleza y en ocasiones realizarse varias referidas a diferentes titulaciones en una misma organización. Cada estudiante o egresado que realiza prácticas tiene tanto un tutor en la organización como un tutor en la Universidad, siendo éste último un profesor de la Universidad de Zaragoza que garantice que las actividades realizadas son apropiadas para la titulación. Al finalizar las prácticas, el tutor de la empresa cumplimenta un cuestionario en el que evalúa al estudiante en una serie de competencias clave y en el que indica si se ha decidido contratar al estudiante o no. De este cuestionario se hablará más extensamente más adelante al ser parte de nuestro estudio empírico.

Como muestra de la relevancia de este servicio de orientación en lo referido a prácticas en organizaciones, en el año 2015 un total de 3.938 estudiantes matriculados en la Universidad de Zaragoza ( $22 \%$ del total) se inscribieron en UNIVERSA, solicitando orientación y/o prácticas en empresas e instituciones. De ellos, un $68 \%$ realizaron dichas prácticas. Así pues, esta importante demanda viene a confirmar la necesaria existencia de este tipo de servicios como apoyo a los jóvenes universitarios en su desarrollo profesional (UNIVERSA, 2015). 


\section{El presente estudio}

Dada la relevancia de las competencias genéricas para el desempeño laboral, resulta clave la realización de investigaciones que contribuyan a favorecer debidamente el desarrollo de dichas competencias en el ámbito universitario. En este sentido, consideramos pertinente estudiar las particularidades existentes en cada Universidad, dado que éstas se desenvuelven en contextos autonómicos diferentes y es el mercado regional el que suele absorber a la mayor parte de titulados. Ante la ausencia de estudios recientes en Aragón, se ha llevado a cabo una investigación empírica a partir de la información existente sobre los estudiantes que realizan prácticas en UNIVERSA.

Nuestro objetivo es determinar las competencias genéricas que muestran, de una forma u otra, estar relacionadas con la obtención de un empleo entre los titulados universitarios en Aragón. Asimismo, queremos explorar si existen diferencias en dichas competencias respecto al sexo, dado que como indica Morley (2001) éste suele olvidarse a la hora de analizar la empleabilidad en la educación superior y, además, ha mostrado ser una variable a tener en cuenta en la investigación en muy diversos aspectos psicosociales del ámbito laboral (e.g. Escartín, Salin y RodríguezCarballeira, 2013; García-Izquierdo, Moscoso y Ramos-Villagrasa, 2012; Mazei et al., 2015).

\section{MÉTODO}

\section{Participantes}

Participaron en nuestro estudio 1.174 estudiantes de último curso o recién egresados (62\% mujeres, todos ellos menores de 30 años), pertenecientes a los distintos estudios que se imparten en la Universidad de Zaragoza, que han realizado prácticas curriculares y extracurriculares en empresas e instituciones en los años 2014 y 2015, gestionadas por el Servicio de Orientación y Empleo de la Universidad de Zaragoza, UNIVERSA. De los 1.174 participantes, $56(4,77 \%)$ fueron contratados por la empresa en la que realizaron las prácticas, en 1.095 casos (93,27\%) se recomendó su contratación, a pesar de que la empresa no pudiera contratarles en ese momento, y a $23(1,96 \%)$ no se les recomendó su contratación. 


\section{Instrumentos}

La evaluación de los diferentes participantes se realizó a partir del cuestionario de evaluación de las prácticas elaborado por UNIVERSA. Dicho cuestionario es cumplimentado por el tutor de las prácticas de la empresa, quien evalúa al estudiante o recién egresado en 22 aspectos o variables diferentes en escala tipo Likert de 1 a 5 , siendo 1 el valor más bajo y 5 el más alto. Los aspectos a evaluar se encontraban organizados de la siguiente forma: (1) valoración profesional, que comprende: conocimientos, aprendizaje, rendimiento, responsabilidad, autonomía, rapidez y agilidad mental, y calidad del trabajo; (2) habilidades sociales, que comprende: comunicación y fluidez verbal, negociación, trabajo en equipo, cortesía y amabilidad, y colaboración; y (3) valoración personal, dentro de la cual se incluyen las siguientes competencias: seguridad en sí mismo y madurez, constancia, flexibilidad, ambición, entusiasmo y vitalidad, iniciativa, disciplina, asistencia y puntualidad, y presencia física. Finalmente, se incluye un apartado sobre la valoración global de las prácticas (de 1 a 10 puntos) y de expectativas futuras de contratación (si piensa contratar o no al estudiante y si, en caso de no poder hacerlo, recomendaría o no su contratación).

De todas las áreas analizadas, solamente conocimientos y rendimiento pueden ser consideradas competencias específicas, siendo el resto competencias genéricas. Por tanto, resulta pertinente para los fines de nuestro estudio.

\section{Procedimiento}

Para la consecución de los objetivos planteados se comenzó recopilando en una base de datos todos los cuestionarios. Después, esta información se analizó mediante el programa de análisis estadístico SPSS v.21.

\section{Análisis}

La información recogida ha sido analizada utilizando estadística descriptiva (media, desviación típica, valores mínimos y máximo) y análisis de diferencia de medias mediante prueba $T$. 


\section{Resultados}

A continuación, se presentan los resultados de nuestro estudio. En primer lugar, la Tabla 1 muestra los estadísticos descriptivos de las diferentes competencias analizadas.

Tabla 1. Estadísticos descriptivos

\begin{tabular}{|c|c|c|c|c|}
\hline Variable & M & $D T$ & Valor mínimo & Valor máximo \\
\hline Conocimientos & 4,23 & 0,72 & 2 & 5 \\
\hline Aprendizaje & 4,60 & 0,63 & 2 & 5 \\
\hline Rendimiento & 4,46 & 0,71 & 1 & 5 \\
\hline Responsabilidad & 4,63 & 0,64 & 1 & 5 \\
\hline Autonomía & 4,27 & 0,79 & 1 & 5 \\
\hline Rapidez y agilidad mental & 4,50 & 0,66 & 2 & 5 \\
\hline Calidad del trabajo & 4,49 & 0,66 & 2 & 5 \\
\hline Comunicación y fluidez verbal & 4,45 & 0,71 & 1 & 5 \\
\hline Negociación & 4,22 & 0,84 & 1 & 5 \\
\hline Trabajo en equipo & 4,59 & 0,62 & 1 & 5 \\
\hline Cortesía y amabilidad & 4,68 & 0,56 & 1 & 5 \\
\hline Colaboración & 4,68 & 0,56 & 1 & 5 \\
\hline Seguridad en sí mismo y madurez & 4,31 & 0,74 & 1 & 5 \\
\hline Constancia & 4,49 & 0,66 & 1 & 5 \\
\hline Flexibilidad & 4,50 & 0,67 & 1 & 5 \\
\hline Ambición & 4,25 & 0,83 & 1 & 5 \\
\hline Entusiasmo y vitalidad & 4,49 & 0,70 & 1 & 5 \\
\hline Iniciativa & 4,30 & 0,81 & 1 & 5 \\
\hline Disciplina & 4,64 & 0,58 & 1 & 5 \\
\hline Asistencia y puntualidad & 4,75 & 0,54 & 1 & 5 \\
\hline Presencia física & 4,76 & 0,50 & 1 & 5 \\
\hline$N=1.174$ & & & & \\
\hline
\end{tabular}


Como puede verse en dicha tabla, en general las valoraciones que reciben los participantes son elevadas, con una media superior a 4 en todos los casos y desviaciones menores a la unidad (entre 0,50 y 0,84). También se observa que en todas las variables al menos un participante alcanza el valor máximo, y que en las variables conocimientos, aprendizaje, rapidez y agilidad mental, y calidad del trabajo ningún participante obtiene una puntuación inferior a 2.

Como ya se ha mencionado, para el objetivo de este artículo resulta de interés analizar el perfil de los participantes que son contratados respecto a los que no lo son, y aquéllos que reciben una recomendación desfavorable (desaconsejar su contratación) frente a los que no la reciben. De esta forma, nos centramos en analizar las particularidades de los participantes incluidos en las condiciones más extremas, que son las que revisten interés (qué condiciones son las que favorecen la contratación o desaconsejan la contratación a otras empresas). Así pues, se procedió al análisis de diferencias de medias entre los participantes. Los resultados, que se muestran en las tablas 2 y 3 , indican que entre los trabajadores contratados y los que no lo han sido existen diferencias en rendimiento $(t=-3,36, p \leq .01)$, autonomía $(t=-2,45$, $p \leq .05)$, seguridad en uno mismo y madurez $(t=-2,12, p \leq .05) \mathrm{e}$ iniciativa ( $t=-2,07, p \leq .05)$, todas ellas a favor de los contratados, mientras que aquéllos cuya contratación no se recomienda obtienen puntuaciones significativamente más bajas en todas las competencias analizadas.

\section{Tabla 2. Diferencia de medias entre participantes no contratados $(n=1.118)$ y contratados $(n=56)$}

\begin{tabular}{|c|c|c|c|}
\hline Variable & $\boldsymbol{M}_{\boldsymbol{n}}$ & $\boldsymbol{M}_{\boldsymbol{c}}$ & $\boldsymbol{t}$ \\
\hline Conocimientos & 4,23 & 4,29 & $-0,66$ \\
\hline Aprendizaje & 4,60 & 4,73 & $-1,55$ \\
\hline Rendimiento & 4,45 & 4,70 & $-3,36^{\star \star}$ \\
\hline Responsabilidad & 4,63 & 4,79 & $-1,83$ \\
\hline Autonomía & 4,26 & 4,48 & $-2,45^{\star}$ \\
\hline
\end{tabular}


Competencias clave para la empleabilidad de los egresados universitarios:

Un análisis en la Universidad de Zaragoza

\begin{tabular}{|c|c|c|c|}
\hline Rapidez y agilidad mental & 4,49 & 4,66 & $-1,94$ \\
\hline Calidad del trabajo & 4,49 & 4,55 & $-0,81$ \\
\hline Comunicación y fluidez verbal & 4,44 & 4,59 & $-1,45$ \\
\hline Negociación & 4,22 & 4,25 & $-0,22$ \\
\hline Trabajo en equipo & 4,58 & 4,70 & $-1,34$ \\
\hline Cortesía y amabilidad & 4,68 & 4,73 & $-0,87$ \\
\hline Colaboración & 4,68 & 4,82 & $-1,90$ \\
\hline Seguridad en sí mismo y madurez & 4,31 & 4,48 & $-2,12^{*}$ \\
\hline Constancia & 4,50 & 4,55 & $-0,57$ \\
\hline Flexibilidad & 4,50 & 4,55 & $-0,56$ \\
\hline Ambición & 4,25 & 4,36 & $-1,10$ \\
\hline Entusiasmo y vitalidad & 4,48 & 4,64 & $-1,66$ \\
\hline Iniciativa & 4,29 & 4,52 & $-2,07^{*}$ \\
\hline Disciplina & 4,64 & 4,75 & $-1,43$ \\
\hline Asistencia y puntualidad & 4,75 & 4,77 & $-0,20$ \\
\hline Presencia física & 4,76 & 4,82 & $-1,11$ \\
\hline
\end{tabular}


Tabla 3. Diferencia de medias entre participantes no recomendados $(n=23)$ y los recomendados o contratados $(n=1.151)$

\begin{tabular}{|c|c|c|c|}
\hline Variable & $M_{n r}$ & $M_{r}$ & $t$ \\
\hline Conocimientos & 3,61 & 4,24 & $-3,59^{\star \star}$ \\
\hline Aprendizaje & 3,56 & 4,63 & $-8,21^{\star * *}$ \\
\hline Rendimiento & 3,17 & 4,48 & $-9,09^{* * *}$ \\
\hline Responsabilidad & 3,57 & 4,65 & $-8,31^{\star \star *}$ \\
\hline Autonomía & 3,17 & 4,29 & $-6,86^{\star \star *}$ \\
\hline Rapidez y agilidad mental & 3,73 & 4,51 & $-5,64^{* \star *}$ \\
\hline Calidad del trabajo & 3,61 & 4,51 & $-6,74^{\star \star \star}$ \\
\hline Comunicación y fluidez verbal & 3,43 & 4,47 & $-7,06^{\star \star \star}$ \\
\hline Negociación & 3,43 & 4,24 & $-4,62^{\star \star \star}$ \\
\hline Trabajo en equipo & 3,52 & 4,61 & $-8,60^{\star \star *}$ \\
\hline Cortesía y amabilidad & 3,91 & 4,70 & $-6,71^{\star \star \star}$ \\
\hline Colaboración & 3,78 & 4,70 & $-7,93^{\star \star *}$ \\
\hline Seguridad en sí mismo y madurez & 3,56 & 4,33 & $-4,98^{\star \star \star}$ \\
\hline Constancia & 3,57 & 4,52 & $-6,95^{\star \star \star}$ \\
\hline Flexibilidad & 3,39 & 4,53 & $-8,15^{\star \star \star}$ \\
\hline Ambición & 3,43 & 4,27 & $-4,81^{\star \star \star}$ \\
\hline Entusiasmo y vitalidad & 3,35 & 4,51 & $-8,13^{\star \star \star}$ \\
\hline Iniciativa & 3,26 & 4,32 & $-6,29^{* \star *}$ \\
\hline Disciplina & 3,78 & 4,70 & $-7,34^{\star \star \star}$ \\
\hline Asistencia y puntualidad & 4,00 & 4,77 & $-6,86^{\star \star \star}$ \\
\hline Presencia física & 4,34 & 4,77 & $-4,03^{\star \star \star}$ \\
\hline
\end{tabular}

Por último, se exploraron las diferencias según sexo entre todas las competencias, encontrándose que solamente la referida 
a presencia física era significativa $(t=-4,23, p=.001)$, siendo superior la media alcanzada por las mujeres $(4,81)$ que por los hombres $(4,69)$.

\section{DISCUSIÓN}

La investigación aquí realizada tenía como objetivo determinar qué competencias genéricas mostraban, de una forma $u$ otra, estar relacionadas con la obtención de un empleo entre los titulados universitarios de Aragón. En este sentido, los resultados de nuestro estudio indican que las competencias que contribuyen a la empleabilidad son el rendimiento, la autonomía, la seguridad en uno mismo y madurez, y la iniciativa. De estas competencias, solamente rendimiento es una competencia específica, siendo el resto competencias genéricas. Además, las citadas competencias genéricas están relacionadas con la capacidad de desenvolverse en el trabajo sin supervisión y adaptándose a las circunstancias cambiantes de la organización, algo cada vez más demandado en el ámbito laboral (MarquesQuinteiro et al., 2015). No es de extrañar, por tanto, que éstas sean las competencias que marcan una diferencia entre quiénes son contratados y no contratados, ya que por la propia metodología docente típica de la Universidad podemos suponerles a todos los participantes un nivel razonablemente similar de otras competencias relevantes como trabajo en equipo o comunicación y fluidez verbal. Sin embargo, sí nos resulta llamativo que no existan diferencias en otras competencias como disciplina, constancia o cortesía y amabilidad; por su contenido, estas competencias parecen estar relacionadas con factores de personalidad, que junto a la aptitud cognitiva son los principales determinantes del desempeño laboral (Ramos-Villagrasa, García-Izquierdo y Navarro, 2013). En cualquier caso, este resultado ofrece algunos indicios sobre qué aspectos deben trabajarse con los estudiantes universitarios y recién egresados, sea desde la formación académica o en el acompañamiento posterior realizado por servicios de orientación y empleo como UNIVERSA. A nuestro juicio, para conseguir este objetivo puede ser especialmente interesante disponer de una evaluación precisa de cada estudiante o recién egresado en dichas competencias clave, en términos de comportamientos de manera que pueda 
plantearse cuál es su nivel real de ejecución y qué carencias tiene que puedan mejorarse mediante formación.

Además, es destacable que los participantes cuya contratación se desaconseja puntúen significativamente por debajo en todas las competencias. Dado que el número de participantes en esta condición es muy bajo $(n=23)$ no podemos descartar la presencia del efecto halo (e.g. Nisbett y DeCamp, 1977), valorando de manera más baja todas las competencias por el escaso desempeño detectado en el participante. Esta es una cuestión que la investigación futura debería aclarar.

También es interesante reseñar que la única competencia en la que se han encontrado diferencias entre mujeres y hombres es en la presencia física. Aunque este resultado puede ser explicado a causa de los estereotipos de género (i.e. se espera que las mujeres cuiden más su imagen personal), consideramos que en el marco de nuestro estudio tiene un carácter anecdótico ya que no ha mostrado ser un factor determinante para la obtención de empleo por parte de los participantes.

\section{Limitaciones y sugerencias de investigación futura}

Como cualquier otra investigación, el presente trabajo adolece de algunas limitaciones que es preciso reconocer. La principal de ellas es la naturaleza del cuestionario empleado en nuestro estudio. Aunque se trata de un instrumento empleado para valorar las prácticas de los estudiantes y recién egresados, se trata de un cuestionario elaborado ad hoc cuya construcción no se apoya en los estándares de medición psicológica (e.g. las directrices internacionales para el uso de los test de la Comisión Internacional de Test, la norma ISO 10667 para la evaluación de personas en entornos laborales contextos organizacionales). Como consecuencia, no existen evidencias acerca de que mide con precisión e incluso de que mide realmente competencias, es decir, no ofrece garantías de fiabilidad y validez (Muñiz, 1992). Además, es posible que exista sesgo de benevolencia (Aguinis, 2013), es decir, la tendencia de los tutores de prácticas a valorar de manera generalmente positiva a la persona en prácticas. Todo esto hace que debamos considerar los resultados como meras orientaciones que deberán ser refrendadas mediante la investigación futura. 
Desafortunadamente, esto no debe ser tomado como un caso aislado, sino como algo habitual seguramente debido al considerable volumen de trabajo que los servicios de orientación han asumido desde su creación, especialmente desde la Crisis Económica. Esto es un ejemplo más de la separación existente entre la investigación científica y la práctica profesional apuntada por García-Izquierdo, Aguinis y Ramos-Villagrasa (2010), a saber: la investigación psicológica ha mostrado la existencia de técnicas para desarrollar pruebas adecuadas con las que medir constructos como las competencias. Sin embargo, en la práctica profesional se utilizan instrumentos sin evidencias de fiabilidad y validez $y$, por tanto, no recomendables para la toma de decisiones. En este sentido, nuestra sugerencia para la investigación futura de la empleabilidad de los universitarios en España es la de realizar los siguientes pasos: (1) revisar la bibliografía científica sobre el tema; (2) identificar las competencias genéricas clave y definirlas en términos conductuales, de manera que puedan establecerse diferentes niveles de competencia; (3) desarrollar una prueba con garantías psicométricas (fiabilidad y validez), basada en anclajes conductuales (Schwab, Heneman, y DeCotiis, 1975), que permita valorar de forma más adecuada competencias específicas del estudiante o egresado en prácticas y, de esta forma, definir necesidades de formación que los servicios de orientación puedan satisfacer; y (4) adaptar al castellano otros instrumentos que puedan resultar útiles a los servicios de orientación, como por ejemplo la escala de percepción de empleabilidad de Rothwell y Arnold (2007).

Junto a ello, otra limitación es la generalización de los resultados. Por una parte, la Universidad de Zaragoza no es la única universidad en Aragón, por lo que no podemos garantizar que su perfil sea generalizable a todos estudiantes y egresados de la comunidad autónoma, o a las del resto de España. No obstante, la Universidad de Zaragoza aglutina al mayor número de estudiantes de Aragón y es una de las principales Universidades españolas, por lo que seguimos considerando de interés los resultados aquí reportados.

A modo de conclusión, consideramos que el presente estudio contribuye a poner en valor el trabajo realizado por los servicios de orientación en las Universidades españolas y resulta un primer paso hacia un proceso de evaluación de prácticas más riguroso 
que pueda incrementar la empleabilidad de los egresados universitarios.

\section{REFERENCIAS}

Aguinis, H. (2013). Performance management. Upper Saddle River, Nueva Jersey: Pearson.

Boyatzis, R. (1982). The competent manager: a model for effective performance. Nueva York: Wiley.

CEDEFOP (2014). Desajuste de competencias: más de lo que parece a simple vista. Disponible en: http://www.cedefop.europa.eu/EN/Files/9087 es.pdf

Escartín, J., Salin, D., y Rodríguez-Carballeira, A. (2013). El acoso laboral o mobbing: similitudes y diferencias de género en su severidad percibida. Revista de Psicología Social, 28, 211224. doi: 10.1174/021347413806196735

Forrier, A., Sels, L., y Stynen, D. (2009). Career mobility at the intersection between agent and structure: A conceptual model. Journal of Occupational and Organizational Psychology, 82, 739-759. doi: 10.1348/096317909X470933

Freire, M. J. (2008). Competencias profesionales de los universitarios. A Coruña: Consejo Social de la Universidad de la Coruña.

García, L. (2008). ¿Qué demandan las empresas de los titulados universitarios? Análisis desde la perspectiva del empresariado sobre el empresariado sobre el proceso de selección de los jóvenes titulados y sus competencias. La Laguna: Fundación Canaria Empresa Universidad de la Laguna.

García-Izquierdo, A. L., Aguinis, H., y Ramos-Villagrasa, P. J. (2010). Science-practice gap in e-recruitment. International Journal of Selection and Assessment, 18, 432-438. doi: 10.1111/J.1468-2389.2010.00525.X.

García-Izquierdo, A. L., García-Izquierdo, M., y Ramos-Villagrasa, P. J. (2007). Aportaciones de la inteligencia emocional y la autoeficacia: Aplicaciones para la selección de personal. Anales de Psicología, 23, 231-239.

García-Izquierdo, A. L., Moscoso, S., y Ramos-Villagrasa, P. J. (2012). Reactions to the fairness of promotion methods: Procedural justice and job satisfaction. International 
Journal of Selection and Assessment, 20, 394-403. doi: 10.1111/ijsa.12002

González, V. y González, R. M. (2008). Competencias genéricas y formación profesional: un análisis desde la docencia universitaria. Revista Iberoamericana de Educación, 47, 185-209.

Goodwin, G. F., Burke, C. S., Wildman, J. y Salas, E. (2008). Team effectiveness in complex organizations. En Salas, E., Goodwin, G. F., \& Burke, C. S. (Eds). Team effectiveness in complex organizations: Cross-disciplinary perspectives and approaches. NY: USA. Routledge.

Herrera, L. H. (2006). El futuro de la psicopedagogía en el marco de la Convergencia Europea de Educación Superior. En M.A. Gallardo, J.A. Fuentes, L. Herrera, S. Rodríguez, G. Rojas, D. Seijo, J.L. Villena y A.M. Fernández (Coords.), I Jornadas de Psicopedagogía: Evaluación e Intervención en Contextos Educativos (pp. 1-13). Granada: Proyecto de Innovación Docente "Plan de Mejora y Evaluación del Prácticum de Psicopedagogía en Melilla".

Hué, C. (2008). El marco de referencia de la nueva Educación Superior. En ICE de la Universidad de Zaragoza (Coord.), Competencias genéricas y transversales de los titulados universitarios. Disponible en http://www.unizar.es/ice/images/stories/ publicacionesICE/Col.\%20Documentos\%2008.pdf

Lantarón, B. S. (2013). Servicios de orientación profesional universitarios: estudio descriptivo. Revista de Docencia Universitaria, 11, 27-42.

Marques-Quinteiro, P., Ramos-Villagrasa, P.J., Passos, A., y Curral, L. (2015). Measuring adaptive performance in individuals and teams. Team Performance Management: An International Journal, 21, 339-360. doi: 10.1108/TPM-03-2015-0014.

Mazei, J., Hüffmeier, J., Freund, P. A., Stuhlmacher, A. F., Bilke, L., \& Hertel, G. (2015). A meta-analysis on gender differences in negotiation outcomes and their moderators. Psychological Bulletin, 141, 85-104. doi: 10.1037/a0038184

Morley, L. (2001). Producing new workers: quality, equality and employability in higher education. Quality in Higher Education, 7, 131-138. doi: 10.1080/13538320120060024

Muñiz, J. (1992). Teoría clásica de los test. Madrid: Pirámide. 
Nisbett, R. E., y DeCamp, T. D. (1977). The Halo Effect: evidence for unconscious alteration of judgments. Journal of Personality and Social Psychology, 35, 250-256.

OEEU (2015). Barómetro de empleabilidad y empleo de los universitarios en España, 2015. Disponible en http://www.funciva.org/uploads/ficheros documentos/1457009450 informe oeeu 2015.pdf

Pereda, S. y Berrocal, F. (2001). Técnicas de Gestión de RRH por competencias. Madrid: Editorial Centro de Estudios Ramón Areces.

Ramos-Villagrasa, P. J., García-Izquierdo, A. L., y Navarro, J. (2013). Predicting the dynamic criteria of basketball players: the influence of the Big Five, motivation and job experience. Journal of Work and Organizational Psychology, 29, 29-35. doi: 10.5093/TR2013A5.

Rothwell, A., y Arnold, J. (2007). Self-perceived employability: development and validation of a scale. Personnel Review, 36, 23-41. doi: 10.1108/00483480710716704

Salmerón, H. (2010) Los Servicios de orientación en la Universidad. Procesos de creación y desarrollo. Disponible en http://rabida.uhu.es/dspace/bitstream/handle/10272/3453/ b15760406.pdf?sequence $=1$

Schwab, D. P., Heneman, H. G., y DeCotiis, T. A. (1975). Behaviorally anchored rating scales: A review of the literature. Personnel Psychology, 28, 549-562. doi: 10.1111/j.1744-6570.1975. tb01392.x

Scott-Ladd, B. y Chan, C. C. A. (2008). Using action research to teach students to manage team learning and improve teamwork satisfaction. Active Learning in Higher Education, 9, 231248.

Tuning (s.f.). Una introducción a Tuning Educational Structures in Europe. La contribución de las universidades al proceso de Bolonia. Disponible en http://www.unideusto.org/tuningeu/ images/stories/documents/General Brochure Spanish version.pdf

Valero, F. J. (2012). Demandas del Mercado laboral a los titulados universitarios. Granada: Vicerrectorado de Estudiantes de la Universidad de Granada. 
UNIVERSA (2015). Memoria 2015. Disponible en http://www. unizar.es/universa/wp-content/uploads/2011/02/MEMORIA 2015 WEB.pdf

Yorke, M. (2006). Employability in higher education: what it is what it is not. York, UK: The Higher Education Academy. 